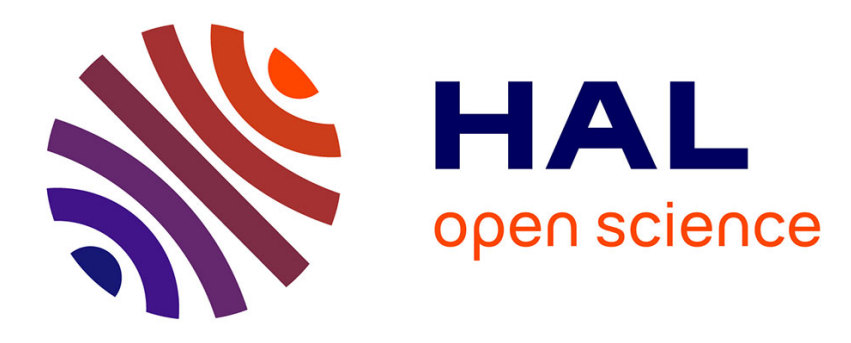

\title{
Quantum Fluctuation Relations for the Lindblad Master Equation
}

Raphael Chetrite, Kirone Mallick

\section{To cite this version:}

Raphael Chetrite, Kirone Mallick. Quantum Fluctuation Relations for the Lindblad Master Equation. Journal of Statistical Physics, 2012, 148, pp.480-501. 10.1007/s10955-012-0557-z • hal-00762245

\section{HAL Id: hal-00762245 \\ https://hal.science/hal-00762245}

Submitted on 6 Dec 2012

HAL is a multi-disciplinary open access archive for the deposit and dissemination of scientific research documents, whether they are published or not. The documents may come from teaching and research institutions in France or abroad, or from public or private research centers.
L'archive ouverte pluridisciplinaire HAL, est destinée au dépôt et à la diffusion de documents scientifiques de niveau recherche, publiés ou non, émanant des établissements d'enseignement et de recherche français ou étrangers, des laboratoires publics ou privés. 


\title{
Quantum Fluctuation Relations for the Lindblad Master Equation
}

\author{
R. Chetrite • K. Mallick
}

Received: 6 December 2011 / Accepted: 25 July 2012 / Published online: 3 August 2012

(C) Springer Science+Business Media, LLC 2012

\begin{abstract}
An open quantum system interacting with its environment can be modeled under suitable assumptions as a Markov process, described by a Lindblad master equation. In this work, we derive a general set of fluctuation relations for systems governed by a Lindblad equation. These identities provide quantum versions of Jarzynski-Hatano-Sasa and Crooks relations. In the linear response regime, these fluctuation relations yield a fluctuation-dissipation theorem (FDT) valid for a stationary state arbitrarily far from equilibrium. For a closed system, this FDT reduces to the celebrated Callen-Welton-Kubo formula.
\end{abstract}

Keywords Fluctuation relations · Quantum Markovian process · Fluctuation Dissipation Theorem

\section{Introduction}

Fluctuations in non-equilibrium systems have been shown to satisfy various remarkable relations $[23,24,44,51,56,62,63,69]$ discovered during the last twenty years. These results have lead to fierce discussions concerning the nature of heat, work and entropy, raising the fundamental issue of understanding the interactions between a given system and its environment (e.g., a thermal bath). In the classical realm, these problems have been progressively clarified whereas they are still under investigation in the quantum world.

Historically, quantum fluctuation relations were first studied by Callen and Welton in 1950 [15]. These authors derived a Fluctuation-Dissipation Theorem for a closed quantum system isolated from its environment, initially in thermal equilibrium and suddenly perturbed by a small time-dependent term added to the time-independent Hamiltonian $H$. This approach was further systematized by Kubo [66] in the linear response theory.

R. Chetrite $(\bowtie)$

Laboratoire J. A. Dieudonné, UMR CNRS 6621, Université de Nice Sophia-Antipolis, Parc Valrose, 06108 Nice Cedex 02, France

e-mail: raphael.chetrite@unice.fr

K. Mallick

Institut de Physique Théorique, CEA Saclay, 91191 Gif-sur-Yvette, France 
Since 2000, three main directions have emerged for constructing quantum fluctuation relations which extend the linear response theory of $[15,66]$ and which are quantum analogues to fluctuation relations for classical systems. In the following, we rapidly review these three different routes by emphasizing their goals and differences. We also cite useful articles.

1. In the first approach, initiated in 2000 by Yukawa [98] and continued by Allahverdyan and Nieuwenhuizen [6], a definition for the quantum work operator is introduced but this operator does not obey any fluctuation relation. More precisely, for closed but non-autonomous systems (with time-dependent Hamiltonian $H_{t}$ ), it is proved in [6] that the naive (inclusive) work operator $H_{t}^{H}(t)-H_{0}^{H}(0)$ (where the exponent $H$ stands for Heisenberg picture) does not satisfy any fluctuation relation, unless $\left[H_{0}, H_{t}\right]=0$. This implies the existence of quantum corrections to the Jarzynski relation [20]. The works of Bochkov and Kuzovlev in 1977 [12], and of Stratonovich in 1994 [89] can be viewed as earlier attempts in this direction.

2. The second line of attack was opened by Kurchan and Tasaki in 2000 [68, 95], and was continued by many groups [9, 17, 64, 79, 81, 92-94]. This approach is based on a different definition for the work. The idea, that leads to a transparent physical picture, is to introduce initial and final measurements of the system's energy, according to the quantummechanical measurement postulates. The work (which is viewed as an energy difference) is then a two-point functional obtained by calculating the difference between the results of the two measurements. This definition of the work differs fundamentally from the previous one (see [32, 41] for a comparison) and does satisfy quantum fluctuation relations. The results of this approach are thoroughly reviewed in [19, 43]. In summary, we can say that this definition of the work as a two-point measurement has been applied in the following different contexts:

(i) Closed but non-autonomous systems prepared in a Gibbs state and isolated from their environment during their evolution (which is thus unitary). Kurchan [68] studied the time cyclic case with $H_{t}=H_{T-t}$ ( $T$ being the period) and proved Jarzynski and Crooks relations in this set-up. Tasaki [95] generalized this result to the non-cyclic case. Many groups [9, 79, 81, 92, 93] simplified the theory. In particular, in 2007, Talkner et al. [92] clarified the fact that work, being characterized through a process with two measurements, 'is not an observable' and cannot be represented by any hermitian operator. The link and the difference with the older Bochkov-Kuzovlev approach [12] (inclusive versus exclusive work) was explained by Campisi et al. in [18] and the possibility to perturb the unitary evolution by N-points measurements was studied by Callens et al. in [16]. Finally, a more general relation for a 'quantum generating functional' was derived by Andrieux et al. (see Eq. (12) of [9]).

(ii) The second set-up corresponds to the general case of an open system continuously interacting with its surroundings. This case can be formally reduced to the previous situation considering the system together with its environment to be a global, closed, system $[17,25$, $26,29,34,36,68,79,94]$. The main physical advantage of this approach is that one can use the previous expression of work as a 2-points measurements. But, such a 'holistic' approach, involving both the system and its surroundings, leads to fluctuation relations that are difficult to assess experimentally. Indeed, the environment is usually large and hardly controllable and only the degrees of freedom are experimentally accessible.

(iii) Finally, the study of heat-matter exchange for two systems in contact [10, 43, 61, 64, $85,86]$ can be viewed as a special instance of the previous set-up. Contact between reservoirs at different temperatures and chemical potentials lead to transport of energy and matter. A famous example is electron counting statistics [65] in which small nanoscale electronic devices exchange electrons.

3 . In a third category of works, the system is modeled by an effective master equation at the mesoscopic scale. This approach was pursued in relatively few articles [25, 34, 36, 42, 
98]. The idea is to consider an open system continuously interacting with its environment and to project out the degrees of freedom of the bath to derive an effective dynamics for the system. Then, it is assumed that the reduced dynamics is described by a closed evolution equation for the density matrix of the system. Under some further assumptions [52, 71], this master equation can be brought into a Markovian form known as the Lindblad equation. In the papers [36, 42], the quantum master equation is treated as an effective classical master equation associated to a pure-jump process; this allows the authors to use the concept of pathwise trajectory and the trajectorial definition of entropy production. The classical fluctuation relations can then be applied. This approach [36, 42], albeit very powerful because it rests on the highly developed field of classical fluctuation relations, doesn't provide us with any explicit relation involving quantum observables. Quantities such as entropy or work are defined along effective classical trajectories and their transposition for the original quantum system are not at all obvious (and in fact, the effective classical process is not uniquely defined). A different philosophy, adopted in [25, 34, 98], is to work directly with the quantum master equation. However, in $[25,98]$ the time evolution was discretized in an ad-hoc manner, and in [34] the transition rates were given in an arbitrary way. At a conceptual level, one could object the relevance of the approach 3, valid only at a mesoscopic scale. Since fluctuation relations can be established at the microscopic scale (by the approach 2), the theory at the mesoscopic scale should simply result from the proved microscopic relations. This objection is not valid for two reasons. First, one should recall that even in the classical case, the fluctuation relations were experimentally tested for effective stochastic models, valid only at a mesoscopic scale [37, 74]. A second argument, given by De Roeck in [36], emphasizes the fact that for a mesoscopic quantum system entropy production is not well defined (in contrast to the case of a classical system) and that mesoscopic fluctuation relation can not be obtained by a coarse-graining procedure.

The present work follows the third approach to quantum fluctuation relations. We study the non-equilibrium fluctuations of an effective open quantum system modeled by a Lindblad master equation. The Lindbladian evolution is a non-unitary dynamics for the density matrix $\rho_{t}$ of the system, described by a differential equation with a generator $L_{t}$ (semi-group property). This effective Markovian description is widely used in Quantum Optics [52]. But unlike [25, 34, 36, 42, 98], our goal, here, is to work directly with the continuous time Lindblad equation, to define an associated time-reversed dynamics and to derive fluctuation relations with quantum observables. Therefore the fluctuations relations we obtain stem from structural and symmetry properties of the Lindblad master equation. The key results of the present work, given in Eqs. (50), (51), (22), (39), represent an original contribution to quantum non-equilibrium statistical mechanics.

Our strategy will be to use a suitable deformation of the master equation, which will allow us to prove a generic relation amongst correlation functions, a kind of book-keeping formula which is a quantum analog of Jarzynski-Hatano-Sasa relation and Crooks relation. Furthermore, by a lowest order expansion, we derive a generalized fluctuation-dissipation theorem valid in the vicinity of a quantum non-equilibrium steady state. For the special case of a closed system, our approach retrieves previously known work identities [9, 34, 68, 92, $95,98]$ as well as the quantum equilibrium fluctuation-dissipation theorem [15, 67].

The outline of this work is as follows. In Sect. 2, we recall some basic properties of the Lindblad equation which represents an effective Markov evolution for a system in contact with an environment. We write a formal solution for the Lindblad equation and give the expression of multi-time correlation functions. In Sect. 3, we prove the Quantum JarzynskiHatano-Sasa relation associated with the Lindblad equation. We use this relation to derive 
a steady-state quantum Fluctuation-Dissipation theorem that generalizes the Kubo-CallenWelton formula (which applies only to a closed system in the vicinity of thermal equilibrium). In Sect. 4, we investigate the properties of a given Lindblad evolution under timereversal. This allows us to prove for open quantum systems a general version of the TasakiCrooks relation. Concluding remarks are given in Sect. 5. Technical details are deferred to Appendices A-C.

\section{Master Equation for Quantum Markov Dynamics}

Consider a quantum system $S$ in contact with a thermal reservoir (or environment) $R$. The total system $S+R$ is closed. Its evolution is unitary and the total density matrix evolves according to the quantum Liouville equation governed by the total Hamiltonian $H_{\text {total }}$ which can be broken into three pieces: the Hamiltonian of the (small) system $H_{S}$, the Hamiltonian of the reservoir $H_{R}$ and the interaction Hamiltonian between the system and its environment $H_{I}$. We are interested in the degrees of freedom of $S$ and therefore we would like to have at our disposal an evolution equation for the density matrix $\rho_{t}$ of the quantum system $S$ alone, the degrees of freedom of the reservoir being traced out. Generically, such an equation is non-local in time: the coupling $H_{I}$ induces memory effects. However, under some specific assumptions such as weak coupling $\left(\lambda \rightarrow 0\right.$ and $t \rightarrow \infty$ with the rescaled time $\tau=\lambda^{2} t$ fixed) $[28,31]$ and factorized initial state $[47,72]$, a differential equation of first order with respect to time can be derived for $\rho_{t}$; one must assume that (i) the full system $S+R$ is prepared in a correlation free state; (ii) the reservoir $R$ is large enough so that it has a very short memory time $\tau_{c}$ (more precisely one must have $\tau_{c} \ll \hbar /\left|H_{I}\right|$ where $\left|H_{I}\right|$ is the typical order of magnitude of the interaction $H_{I}$ matrix elements [55]). This second assumption is the crucial Markov hypothesis: when it is fulfilled a memoryless and coarse-grained description of the system $S$ becomes possible. This condition is generally satisfied in Quantum Optics $[27,55]$.

We point out that the Markov assumption can be also justified without using the weak coupling limit but via a 'singular coupling' limit (bath uncorrelated in time) $[38,53]$ which describes well a classical environment or a system far from equilibrium driven by some external device [57]. Another interesting limit is the low density case [39], in which the small parameter $v$ is the density of the gas in the bath and where the limit $t \rightarrow \infty$ is performed with $\tau=v t$ fixed.

There are two main methods to derive the master equation for quantum Markovian dynamics. One way is to make a precise model for the reservoir (typically an infinite set of quantum oscillators) and to eliminate explicitly the environmental degrees of freedom. The Markov approximation can be analyzed and justified precisely [27]. Another possibility is to study the structural properties of the 'quantum map' that carries the density matrix $\rho_{t}$ at time $t$ into the density matrix $\rho_{t+d t}$ at time $t+d t$. Such a map must be linear, hermiticity preserving, trace conserving and positive. In fact, this map lifted to an operator on the total system $S+R$ (for any given environment) must remain positive (this stronger requirement is called complete positivity) $[52,55]$. These physically reasonable conditions are stringent enough to mathematically constrain the possible forms of the evolution equation of a quantum Markov dynamical system [71]. The resulting equation is called a Lindblad equation. Its generic form, Eq. (1), and some of its basic properties are discussed below.

\subsection{Some Properties of the Lindblad Equation}

We consider a quantum system prepared initially with a density matrix $\pi_{0}$. Because of its interactions with its environment, the density matrix of the system, becomes a function of 
time, and will be denoted by $\rho_{t}$. In the present work, we adopt the framework of Quantum Markovian Dynamics. The evolution of $\rho_{t}$ is thus given by a Lindblad master equation, which can be written in the generic form $[5,13,52,55]$ :

$$
\partial_{t} \rho_{t}=-i\left[H_{t}, \rho_{t}\right]+\sum_{i=1}^{I}\left(V_{i} \rho_{t} V_{i}^{\dagger}-\frac{1}{2} V_{i}^{\dagger} V_{i} \rho_{t}-\frac{1}{2} \rho_{t} V_{i}^{\dagger} V_{i}\right) .
$$

On the right-hand side of this equation, the first term $-i\left[H_{t}, X\right]$ is the conservative part where $H_{t}$ is the Hamiltonian of the system that may depend on time. The other terms represent the interactions of the system with its environment (also called the 'bath') and also represent the effect of measurements (i.e. dissipation and (de)coherence effects). The operators $V_{i}$ are called the Kraus operators, they are not necessarily hermitians and may depend explicitly on time. The Kraus number $I$ depends on the system considered. If the system under consideration is closed, all Kraus operators vanish identically, $V_{i} \equiv 0$, and the Lindblad master equation reduces to the quantum version of the Liouville equation. Equation (1) can be written symbolically as

$$
\partial_{t} \rho_{t}=L_{t}^{\dagger} \rho_{t},
$$

where we have introduced the Lindbladian superoperator $L_{t}^{\dagger}$ which acts on the density matrix $\rho_{t}$ and generates its time-dynamics. We emphasize that $L_{t}^{\dagger}$ is a superoperator because it is a linear map in the space of operators. The fact that we have used in Eq. (2) the symbol $L_{t}^{\dagger}$ for the Lindbladian rather than the more usual notation $L_{t}$ is purely a matter of convention: this will allow us to write some expressions of time-ordered correlations of observables in a simpler manner (because $L_{t}^{\dagger}$ acts on the density matrix $\rho_{t}$ and its conjugate superoperator $L_{t}$ acts on observables). More precisely, the space of operators is endowed with the following Hilbert-Schmidt scalar product $(Y, X)=\operatorname{Tr}\left(Y^{\dagger} X\right)$, where $X$ and $Y$ are arbitrary operators and $Y^{\dagger}$ is the hermitian conjugate of $Y$. This allows us to define a pair of adjoint superoperators $L_{t}$ and $L_{t}^{\dagger}$ as follows

$$
\left(Y, L_{t} X\right)=\operatorname{Tr}\left(Y^{\dagger}\left(L_{t} X\right)\right)=\left(L_{t}^{\dagger} Y, X\right)=\operatorname{Tr}\left(\left(L_{t}^{\dagger} Y\right)^{\dagger} X\right) .
$$

A simple calculation allows us to write

$$
L_{t} X=i\left[H_{t}, X\right]+\sum_{i=1}^{I}\left(V_{i}^{\dagger} X V_{i}-\frac{1}{2} V_{i}^{\dagger} V_{i} X-\frac{1}{2} X V_{i}^{\dagger} V_{i}\right) .
$$

Two important properties of the Lindbladian $L_{t}$ are $L_{t} 1=0$ (Trace conservation) and $L_{t}\left(X^{\dagger}\right)=\left(L_{t} X\right)^{\dagger}$.

The Lindblad equation is extensively used in Quantum Optics. A simple example is a two-level atom emitting a photon in free space. The density matrix $\rho_{t}$ is a 2 by 2 matrix and the Kraus operators reduce to Pauli lowering and rising operators. The Lindblad equation is then simply a set of four coupled first order differential equations [55]. The physical relevance of a non-stationary Lindblad generator with an explicit time-dependence of the Hamiltonian and of the Kraus operators, is justified in [4] if this dependence is slow with respect to the relaxation time of the bath.

\subsection{A Formal Solution of the Lindblad Equation}

The quantum Master equation (2) can be solved formally by introducing the evolution superoperator $P_{0}^{t}$ : 


$$
\rho_{t}=\left(P_{0}^{t}\right)^{\dagger} \pi_{0},
$$

where $\pi_{0}$ represents the density-matrix at the initial time. The evolution superoperator $P_{s}^{t}$ between the two times $s \leq t$ is defined by

$$
P_{s}^{t}=\overrightarrow{\exp }\left(\int_{s}^{t} d u L_{u}\right)=1+\sum_{n=1}^{\infty} \int_{s \leq t_{1} \leq t_{2} \leq \cdots \leq t_{n} \leq t} \prod_{i=1}^{n} d t_{i} L_{t_{1}} L_{t_{2}} \ldots L_{t_{n}} .
$$

In this time-ordered exponential, time is increasing from left to right. This symbolic writing will be very useful to perform formal calculations and to write perturbative expansions. Let us recall how Eq. (5) is proved. First, we observe that this equation is true at $t=0$ because $P_{0}^{0}$ is the identity operator. Then, from the time-ordered exponential (6), we find $\frac{d}{d t} P_{s}^{t}=P_{s}^{t} L_{t}$. This leads us finally to

$$
\frac{d}{d t} \rho_{t}=\left(L_{t}^{\dagger}\left(P_{0}^{t}\right)^{\dagger}\right) \pi_{0}=L_{t}^{\dagger} \rho_{t} .
$$

Thus, $\rho_{t}$ satisfies the Lindblad equation (2) with initial condition $\pi_{0}$. We note that this technique of proving an identity between operators, such as Eq. (5), by showing that both operators are solutions of the same (first order) differential equation with the same initial condition, will be used repeatedly in this work.

\subsection{Expression for Multi-time Correlations}

Using the evolution operator, we can write a general expression for multi-time correlations of different observables. For $0 \leq t_{1} \leq t_{2} \leq \cdots \leq t_{N} \leq t$, the time-ordered correlation of observables $O_{0}, O_{1}, O_{2}, \ldots, O_{N}$ is given by

$$
\left\langle O_{1}\left(t_{1}\right) O_{2}\left(t_{2}\right) \ldots O_{N}\left(t_{N}\right)\right\rangle_{\pi_{0}}=\operatorname{Tr}\left(\pi_{0} P_{0}^{t_{1}} O_{1} P_{t_{1}}^{t_{2}} O_{2} \ldots P_{t_{N-1}}^{t_{N}} O_{N}\right) .
$$

A justification of this expression can be found in [52] or in [13]: One starts by the correlation of two operators at two different times. This correlation can be evaluated in the Heisenberg representation by using the full Hamiltonian of the system plus its environment. In order to obtain an expression that refers to the system alone, the partial trace over the environment has to be performed. Using the assumption that the total density matrix factorizes at each observation time $t_{1}, t_{2}, \ldots, t_{N}$, and the weak coupling assumption that lead to the Lindblad Master equation (2), one shows that the time-ordered two-time correlation function satisfies an evolution equation which is the dual of Eq. (2), i.e. it is governed by the Lindblad operator $L_{t}$. This proves the formula (8) for $N=2$. The general case is then obtained by induction. Besides, a rigorous proof of the formula (8) is given in [11] for a toy model of one harmonic oscillator in a bath of independent harmonic oscillators.

We recall that, in the formula (8), the operator $\pi_{0}$ represents the initial density matrix of the system and emphasize that the superoperator $P_{t_{i}}^{t_{i+1}}$ operates on all the terms to its right. For example, for $N=3$, the explicit expression, with all the required brackets, is given by:

$$
\left\langle O_{1}\left(t_{1}\right) O_{2}\left(t_{2}\right) O_{3}\left(t_{3}\right)\right\rangle_{\pi_{0}}=\operatorname{Tr}\left(\pi_{0} P_{0}^{t_{1}}\left(O_{1} P_{t_{1}}^{t_{2}}\left(O_{2} P_{t_{2}}^{t_{3}}\left(O_{3}\right)\right)\right)\right) \text {. }
$$

\subsection{The Accompanying Density Matrix}

We suppose that the initial density matrix $\pi_{0}$ of the system satisfies $L_{0}^{\dagger} \pi_{0}=0$. For example, the system is prepared in a thermal state at temperature $T=\frac{1}{k \beta}$, where $k$ is Boltzmann's constant and its initial density matrix is $\pi_{0}=Z_{0}^{-1} \exp (-\beta H(0))$. If the system is closed 
and the Hamiltonian is constant in time, then the density matrix does not vary with time: $\pi_{t}=\pi_{0}$. However, if the Hamiltonian $H_{t}$ changes with time and/or if the interactions with the environment are taken into account, the density matrix evolves according to Eq. (2) and the system is out of equilibrium. In particular, at time $t$, the system does not lie in the kernel of the time-dependent generator $L_{t}^{\dagger}$. For example, at time $t$, a closed system with timedependent Hamiltonian $H_{t}$, is not in the Gibbs state $Z_{t}^{-1} \exp \left(-\beta H_{t}\right)$. (The same feature is also true, of course, in classical mechanics.)

Nevertheless, given a time-dependent Lindbladian $L_{t}$, it is useful, following [54], to associate to it the accompanying density-matrix $\pi_{t}$ that satisfies $L_{t}^{\dagger} \pi_{t}=0$. Physically, $\pi_{t}$ represents the stationary state in a system where time is frozen at its instantaneous value $t$. However, as we emphasized above, at time $t$ the true density matrix of the system differs from the accompanying density-matrix: $\rho_{t} \neq \pi_{t}$. The reason is that $\pi_{t}$ depends on time, and therefore it can not satisfy Eq. (2):

$$
\frac{d}{d t} \pi_{t} \neq 0 \quad \text { whereas } L_{t}^{\dagger} \pi_{t}=0 .
$$

For a closed system, we have $\pi_{t}=Z_{t}^{-1} \exp \left(-\beta H_{t}\right)$. This form also holds for a Markov process weakly coupled with a thermal bath at temperature $\beta^{-1}$ (if the bath satisfies the KMS condition, see $[5,87,88]$ ). In the following, we shall consider the general, far from equilibrium case, where $\pi_{t}$ is not supposed to be given by the canonical Gibbs-Boltzmann formula.

\section{A Quantum Jarzynski-Hatano-Sasa Identity for Lindblad Dynamics}

A key idea that lies at the heart of the Jarzynski identity in the classical case is to consider non-equilibrium averages over weighted trajectories. This crucial feature was clearly recognized and stated in the very early works $[62,63]$. If the weighting factor is chosen to be the exponential of the work performed on the system, then weighted averages along a nonequilibrium process between times 0 and $t$ can be reduced to thermal averages performed with the accompanying Gibbs measure at time $t$. An equivalent formulation due to Hummer and Szabo [60] is to consider an auxiliary system governed by a fictitious dynamics, constructed in such a way that at each time $t$ the auxiliary system lies in the accompanying steady-state measure at time $t$ of the initial system. Thus, averages on the fictitious system can be written as steady-state averages in the initial system. Besides, using the FeynmanKac formula, Hummer and Szabo showed that averages over the fictitious system are given by averages over the initial system weighted by the Jarzynski factor (the exponential of the work).

It is important to note that exactly the same idea of considering a modified dynamics appears in the proof by Kurchan of the Gallavotti-Cohen relation for Langevin dynamics [69] and also in the general derivation of the Gallavotti-Cohen symmetry for Markovian systems by Lebowitz and Spohn [70].

Our aim in this section is to prove a Jarzynski-Hatano-Sasa identity for a quantum Markovian dynamics by using a similar technique: the Lindblad dynamics is deformed so that the accompanying density-matrix $\pi_{t}$ of the initial system becomes the true density-matrix at time $t$ of the fictitious auxiliary system. Then, an operator version of the Feynman-Kac formula will allow us to relate expectations values over the auxiliary system to averages on the initial system, leading us to a quantum version of the Jarzynski-Hatano-Sasa identity. In the case of a closed system, we shall show that this identity is equivalent to relations that were previously known. Finally, we deduce from our general result a fluctuation-dissipation theorem valid for an arbitrary steady state. 


\subsection{A Modified Dynamics for the Accompanying Density Matrix}

The accompanying density-matrix $\pi_{t}$ does not obey the Lindblad equation (2). However, $\pi_{t}$ is a tautological solution of the modified evolution equation

$$
\partial_{t} \pi_{t}=\left(L_{t}+\pi_{t}^{-1}\left(\partial_{t} \pi_{t}\right)\right)^{\dagger} \pi_{t} .
$$

We introduce the non-stationary operator

$$
W_{t}=-\left(\pi_{t}\right)^{-1}\left(\partial_{t} \pi_{t}\right),
$$

which reduces in the classical limit to the rate of injected dissipative power.

Remark In the context of Quantum Markovian system, a concept of average rate of work was introduced in $[4,80]$ as $w_{t}=\operatorname{Tr}\left(\rho_{t} \partial_{t} H_{t}\right)$. However, as in the case of closed systems, this definition does not correspond to the average of an operator that satisfies a fluctuation relation [29]. Therefore, we shall not use it here. The operator $W_{t}$ defined above is not related to $w_{t}$ in general, i.e. $\left\langle W_{t}\right\rangle=\operatorname{Tr}\left(\rho_{t} W_{t}\right) \neq w_{t}$. However, we shall prove that $W_{t}$ does indeed satisfy a fluctuation relation.

We define a modified superoperator as follows

$$
L_{t, 1}=L_{t}+\left(\pi_{t}\right)^{-1}\left(\partial_{t} \pi_{t}\right)=L_{t}-W_{t},
$$

where $W_{t}$ acts on a given density-matrix by a multiplication on the left. This superoperator $L_{t, 1}$ corresponds to the auxiliary dynamics $\partial_{t} \rho_{t}=L_{t, 1}^{\dagger} \rho_{t}$ and allows us to define a modified evolution superoperator $P_{s, 1}^{t}$ as follows:

$$
P_{s, 1}^{t}=\overrightarrow{\exp }\left(\int_{s}^{t} L_{u, 1} d u\right)=\overrightarrow{\exp }\left(\int_{s}^{t}\left(L_{u}+\pi_{u}^{-1}\left(\partial_{u} \pi_{u}\right)\right) d u\right) .
$$

Equation (13) can then be rewritten as

$$
\partial_{t} \pi_{t}=L_{t, 1}^{\dagger} \pi_{t},
$$

and its formal solution is given by

$$
\pi_{t}=\left(P_{0,1}^{t}\right)^{\dagger} \pi_{0}
$$

We observe that this formula for the accompanying matrix is similar to Eq. (5) for the 'true' density matrix of the system.

Consider now an arbitrary observable $A$. Then, using Eq. (14), we can write

$$
\operatorname{Tr}\left(\pi_{0} P_{0,1}^{t} A\right)=\operatorname{Tr}\left(\pi_{t} A\right) .
$$

This identity means that averages for the fictitious evolution which are performed by using the modified evolution superoperator $P_{s, 1}^{t}$ reduce to averages performed with the accompanying density-matrix at time $t$.

\subsection{Proof of the Quantum Jarzynski-Hatano-Sasa Relation}

A quantum version of the Jarzynski-Hatano-Sasa Relation will be obtained by relating the auxiliary evolution superoperator $P_{s, 1}^{t}$ to the initial evolution superoperator $P_{s}^{t}$. To achieve this aim, we need to prove an extension of the Feynman-Kac formula. 
We write a Dyson-Schwinger series expansion for $P_{0,1}^{t}$, considering $W_{t}$ to be a perturbation of the Lindbladian $L_{t}$ :

$$
P_{0,1}^{t}=\sum_{n}(-1)^{n} \int_{0 \leq t_{1} \leq t_{2} \leq \cdots \leq t_{n} \leq t} \prod_{i=1}^{n} d t_{i} P_{0}^{t_{1}} W_{t_{1}} P_{t_{1}}^{t_{2}} W_{t_{2}} \ldots P_{t_{N-1}}^{t_{N}} W_{t_{N}} P_{t_{N}}^{t} ;
$$

we recall that the superoperator $P_{t_{i}}^{t_{i+1}}$ operates on all the terms to its right. This well-known formula [82] can be proved by showing that both sides of the equation satisfy the same differential equation and are identical at $t=0$. Inserting this expansion on the r.h.s. of Eq. (15), we obtain

$$
\begin{aligned}
& \operatorname{Tr}\left(\pi_{0} P_{0,1}^{t} A\right) \\
& \quad=\sum_{n}(-1)^{n} \int_{0 \leq t_{1} \leq t_{2} \leq \cdots \leq t_{n} \leq t} \prod_{i=1}^{n} d t_{i} \operatorname{Tr}\left(\pi_{0} P_{0}^{t_{1}} W_{t_{1}} P_{t_{1}}^{t_{2}} W_{t_{2}} \ldots P_{t_{N-1}}^{t_{N}} W_{t_{N}} P_{t_{N}}^{t} A\right) .
\end{aligned}
$$

Rewriting the trace term inside the integrals as a multi-time correlation function via Eq. (8), we find

$$
\operatorname{Tr}\left(\pi_{0} P_{0,1}^{t} A\right)=\sum_{n}(-1)^{n} \int_{0 \leq t_{1} \leq t_{2} \leq \cdots \leq t_{n} \leq t} \prod_{i=1}^{n} d t_{i}\left\langle W_{t_{1}}\left(t_{1}\right) W_{t_{2}}\left(t_{2}\right) \ldots W_{t_{N}}\left(t_{N}\right) A(t)\right\rangle_{\pi_{0}} .
$$

We note that on the r.h.s. of Eq. (8), the operators $O_{i}$ depend on the time $t_{i}$; this time dependence is written as an argument $O_{i}\left(t_{i}\right)$. Here we have $O_{i}=W_{t_{i}}$ which already depends on time. A supplementary time dependence is introduced through the use of Eq. (8), which now appears as $W_{t_{i}}\left(t_{i}\right)$. By linearity, equation (18) is identical to

$$
\begin{aligned}
& \operatorname{Tr}\left(\pi_{0} P_{0,1}^{t} A\right) \\
& \quad=\left\langle\left\{\sum_{n}(-1)^{n} \int_{0 \leq t_{1} \leq t_{2} \leq \cdots \leq t_{n} \leq t} \prod_{i=1}^{n} d t_{i} W_{t_{1}}\left(t_{1}\right) W_{t_{2}}\left(t_{2}\right) \ldots W_{t_{N}}\left(t_{N}\right)\right\} A(t)\right\rangle_{\pi_{0}} .
\end{aligned}
$$

The term between curly brackets can be resummed as a time-ordered exponential

$$
\operatorname{Tr}\left(\pi_{0} P_{0,1}^{t} A\right)=\left\langle\overrightarrow{\exp }\left(-\int_{0}^{t} W_{u}(u) d u\right) A(t)\right\rangle_{\pi_{0}} .
$$

This formula is an extension of the Feynman-Kac formula for quantum Markov semi-groups. We emphasize that, due to non-commutativity of operators, the exponential that appears in the usual Feynman-Kac formula is replaced here by a time-ordered exponential. Moreover, we remark that, although there exist many generalizations of the Feynman-Kac formulae [1, 30], the present one seems to be original (see [75] for a special case). Finally, using Eq. (15), the following relation is derived:

$$
\operatorname{Tr}\left(\pi_{t} A\right)=\left\langle\overrightarrow{\exp }\left(-\int_{0}^{t} W_{u}(u) d u\right) A(t)\right\rangle_{\pi_{0}} .
$$

This identity is a quantum extension of the classical Jarzynski-Hatano-Sasa identity and is one of the main results of the present work. In particular, if we take $A=\mathbb{1}$ then Eq. (21) becomes

$$
\left\langle\overrightarrow{\exp }\left(-\int_{0}^{t} W_{u}(u) d u\right)\right\rangle_{\pi_{0}}=1
$$


where we use the fact that $\operatorname{Tr}\left(\pi_{t}\right)=1$. Note that if we interpret the mean values as classical averages and the operators as commuting c-numbers, then Eq. (22) reduces to the classical Jarzynski-Hatano-Sasa relation. The relation (22) can be interpreted as a kind of a book-keeping formula which allows us to write identities amongst correlation functions. We emphasize that the operator $W_{t}$ is not Hermitian: this is a signature of the fact that 'Work is not an observable' [92]. However, for the special case of a closed system, the work-term can be written as a product of two observables, as will be shown below, allowing us to retrieve previously known work identities [9, 34, 68, 92, 95, 98]. Besides, from a first order expansion of Eq. (22) we shall derive a generalized fluctuation-dissipation theorem valid in the vicinity of a quantum non-equilibrium steady state.

\subsection{The Case of a Closed Quantum System}

We consider the special case of a closed, isolated system, governed by a time-dependent Hamiltonian. The Lindbladian reduces to the Liouville operator, $L_{t} . X=i\left[H_{t}, X\right]$, and the evolution of the system is unitary. For a closed system, the evolution superoperator $P_{0}^{t}$ acts on an observable $X$ as follows

$$
P_{0}^{t} X=\left(U_{0}^{t}\right)^{\dagger} X U_{0}^{t},
$$

where the unitary evolution operator is defined as

$$
U_{0}^{t}=\overleftarrow{\exp } \int_{0}^{t} d u\left(-i H_{u}\right)
$$

Here, the arrow pointing towards the left over the time-ordered exponential indicates that early times are written on the right and later times on the left.

The image of $X$ by the evolution superoperator $P_{0}^{t}$ defines the Heisenberg operator $X^{\mathcal{H}}(t)$ where the upper-script $\mathcal{H}$ stands for Heisenberg:

$$
P_{0}^{t} X=X^{\mathcal{H}}(t) .
$$

We also note that $P_{0}^{t}$ is a multiplicative superoperator i.e. for any two observables $X$ and $Y$ we have

$$
P_{0}^{t}(X Y)=P_{0}^{t}(X) P_{0}^{t}(Y) .
$$

(This is not true in general for an open system.) Thanks to this property, the r.h.s. of the general expression (8) for multi-time correlations can be evaluated and we obtain:

$$
\left\langle O_{1}\left(t_{1}\right) O_{2}\left(t_{2}\right) \ldots O_{N}\left(t_{N}\right)\right\rangle_{\pi_{0}}=\operatorname{Tr}\left(\pi_{0} O_{1}^{\mathcal{H}}\left(t_{1}\right) O_{2}^{\mathcal{H}}\left(t_{2}\right) \ldots O_{N}^{\mathcal{H}}\left(t_{N}\right)\right) .
$$

If we substitute this expression in Eq. (18) and retrace the steps from Eq. (19) to Eq. (21), we find that for a closed system the quantum Jarzynski-Hatano-Sasa relation can be written as

$$
\operatorname{Tr}\left(\pi_{t} A\right)=\operatorname{Tr}\left(\pi_{0} \overrightarrow{\exp }\left(-\int_{0}^{t} W_{u}(u)^{\mathcal{H}} d u\right) A^{\mathcal{H}}(t)\right),
$$

where from Eqs. (25) and (26), we have

$$
W_{u}(u)^{\mathcal{H}}=-\left(\pi_{u}^{-1}\right)^{\mathcal{H}}(u)\left(\partial_{u} \pi_{u}\right)^{\mathcal{H}}(u) .
$$

We also recall that for a closed system, the accompanying density is given by $\pi_{t}=$ $Z_{t}^{-1} \exp \left(-\beta H_{t}\right)$. Equation (28) can be simplified thanks to the following operator identity, which is proved in Appendix A:

$$
\overrightarrow{\exp }\left(-\int_{0}^{t} W_{u}(u)^{\mathcal{H}} d u\right)=\left(\pi_{0}\right)^{-1} \pi_{t}^{\mathcal{H}}(t) .
$$


Substituting Eq. (30) in Eq. (28), the following (tautological) identity is obtained

$$
\operatorname{Tr}\left(\pi_{t} A\right)=\operatorname{Tr}\left\{\pi_{0}\left(\left(\pi_{0}\right)^{-1} \pi_{t}^{\mathcal{H}}(t)\right) A^{\mathcal{H}}(t)\right\} .
$$

Taking $A=\mathbb{1}$, we end up with the quantum Jarzynski relation for closed systems as first found by Kurchan and Tasaki $[68,95]$ :

$$
\operatorname{Tr}\left(\pi_{0} \exp \left(\beta H_{0}^{\mathcal{H}}(0)\right) \exp \left(-\beta H_{t}^{\mathcal{H}}(t)\right)\right)=\frac{Z_{t}}{Z_{0}} .
$$

Hence, for closed systems, the quantum work $W$ characterizes a process where the energy is measured twice, at time 0 and time $t$ [92].

Remark If we suppose that $H_{u}$ commutes with $\partial_{u} H_{u}$, then the formula (28) can be brought into a simpler form. From Eq. (29), we have $W_{u}^{\mathcal{H}}(u)=-\partial_{u}\left(\ln Z_{u}\right)+\beta\left(\partial_{u} H_{u}\right)^{\mathcal{H}}(u)$ where we explicitly used that $\left[H_{u}, \partial_{u} H_{u}\right]=0$. We then obtain

$$
\frac{Z_{t}}{Z_{0}} \operatorname{Tr}\left(\pi_{t} A\right)=\operatorname{Tr}\left(\pi_{0} \overrightarrow{\exp }\left(-\beta \int_{0}^{t}\left(\partial_{u} H_{u}\right)^{\mathcal{H}}(u) d u\right) A^{\mathcal{H}}(t)\right) .
$$

The case $A=1$ gives the Hänggi-Talkner form [92] of the quantum Jarzynski relation for closed systems:

$$
\operatorname{Tr}\left(\pi_{0} \overrightarrow{\exp }\left(-\beta \int_{0}^{t}\left(\partial_{u} H_{u}\right)^{\mathcal{H}}(u) d u\right)\right)=\frac{Z_{t}}{Z_{0}} .
$$

Remark that this relation is true if $\left[H_{u}, \partial_{u} H_{u}\right]=0$. This fact was neglected in [92] and corrected in an addendum of [19].

\subsection{Steady-State Quantum Fluctuation Dissipation Theorem}

We now return to the general case of an open system. One main advantage of the identity (21) is that it implies a generalized fluctuation-dissipation theorem valid in the vicinity of a quantum non-equilibrium steady state. We start with a Lindbladian $L_{0}$, which does not depend on time, with invariant density-matrix given by $\pi_{0}$. We then consider a perturbation of $L_{0}$ of the form

$$
L_{t}=L_{0}-h^{a}(t) M_{a} .
$$

The time-dependent perturbations $h^{a}(t)$ are supposed to be small and a summation over the repeated index $a$ is understood. At first order, the accompanying density-matrix $\pi_{t}$, that satisfies $L_{t}^{\dagger} \cdot \pi_{t}=0$, is given by

$$
\pi_{t}=\pi_{0}+h^{a}(t) \epsilon_{a},
$$

where $\epsilon_{a}$ satisfies

$$
L_{0}^{\dagger} \cdot \epsilon_{a}=M_{a}^{\dagger} \cdot \pi_{0}
$$

The operator $W_{t}$, defined in (10), reads

$$
W_{t}=-\dot{h}^{a}(t) D_{a} \quad \text { with } D_{a}=\pi_{0}^{-1} \epsilon_{a} .
$$

We now take the functional derivative of the identity (21) w.r.t. $h^{a}(u)$ for $u<t$. The derivative of the 1.h.s. vanishes because $\pi_{t}$ depends only on $h^{a}(t)$ and not on $h^{a}(u)$ for 
$u<t$. We evaluate the derivative of the r.h.s. by using the first order expression (38) for $W_{u}$. This yields

$$
\left.\frac{\delta\langle A(t)\rangle}{\delta h^{a}(u)}\right|_{h=0}=\frac{d}{d u}\left\langle\left. D_{a}(u) A(t)\right|_{\pi_{0}} .\right.
$$

We emphasize that the expectation value on the r.h.s. is taken with respect to the unperturbed density matrix $\pi_{0}$. By choosing $A_{T}=D_{b}(T)$, Eq. (39) becomes structurally similar to the usual equilibrium fluctuation dissipation theorem. This generalizes to the quantum case a result obtained recently for classical systems [22, 84] (see [97] for an alternative approach).

Remark In the case of a closed system perturbed near equilibrium the steady-state quantum Fluctuation Dissipation Theorem (39) reduces to the celebrated Callen-Welton-Kubo relation $[15,67]$. The details of the proof are given in Appendix B.

\section{Time Reversal and a Quantum Tasaki-Crooks Relation}

Symmetry by time reversal lies at the heart of many exact identities in non-equilibrium statistical mechanics: indeed, various fluctuation relations can be derived by comparing the averages performed on a given process with those performed on the time-reversed process. We shall first recall how time reversal can be defined for quantum Markov processes. Then we shall use this operation to derive a general version of the Tasaki-Crooks Relation, valid for open quantum systems.

\subsection{Time-Reversal for Lindblad Dynamics}

In Quantum Mechanics, time reversal on the states $\psi$ of the Hilbert space is implemented by an anti-unitary operator [90] that we denote by $\theta$. The operator $\theta$ is anti-linear and it satisfies $\theta^{2}=1, \theta=\theta^{-1}=\theta^{\dagger}$. For a spin-0 particle without magnetic field, $\theta$ can be identified with the complex conjugation operator (i.e. by time reversal, the Schrödinger wave function $\psi$ becomes $\psi^{*}$ ). In presence of a magnetic field, this time-inversion operation must be supplemented by the requirement that the reversed system evolves with vector potential $A^{R}=-A$. Time reversal of observables (which are operators acting on the Hilbert space of states) is implemented by a superoperator $K$ that acts on an operator $X$ as follows [2, 78]:

$$
K X=\theta X \theta^{-1} \text {. }
$$

The superoperator $K$ is anti-unitary, with $K^{2}=1, K=K^{-1}=K^{\dagger}$ and is multiplicative, i.e. $K(X Y)=K(X) K(Y)$.

We can now define time reversal for a quantum Markov process. We consider the case of a system with a constant Lindbladian $L$ that lies in a steady state with density-matrix $\pi$. Conditions for defining the time-reversed quantum Markov process have been stated by various authors $[2,45,46,78]$. The superoperator $L^{R}$ that governs the reversed process is given by

$$
L^{R}=K \pi^{-1} L^{\dagger} \pi K
$$

where $\pi^{-1}$ and $\pi$ are understood as left-multiplication superoperators, i.e., if $X$ is an operator we have $L^{R}(X)=K\left(\pi^{-1} L^{\dagger}(\pi(K X))\right)$. The condition of micro-reversibility or detailed balance is then expressed as $L^{R}=L$ which is equivalent to $K \pi^{-1} L^{\dagger} \pi K=L$. Note also that this relation and its conjugate imply that $\pi K=K \pi$, so $K(\pi)=\pi$; thus, the detailed 
balance condition also takes the form $L=\pi^{-1} K L^{\dagger} K \pi$ which is identical to the condition given in [91] (relation 4.8). The exponentiation of this last formula leads us to the finite time formula $\pi P_{0}^{T}=K\left(P_{0}^{T}\right)^{\dagger} K \pi$ which can be written as

$$
\operatorname{Tr}\left(B^{\dagger} \pi P_{0}^{T} A\right)=\operatorname{Tr}\left(\left(K A^{\dagger}\right) \pi P_{0}^{T}(K B)\right)
$$

for two arbitrary observables $A$ and $B$. Formula (42) coincides with the definition of a detailed balance given by Majewski [78] inspired by Agarwal (relation 2.19 of [2]) and by Fagnola et al. [46], which give a characterization of the Lindbladians that satisfy detailed balance. Besides, we must underline that there exists still another definition of quantum detailed balance in the sense of Frigerio et al. [49, 50] and Alicki [3] which can be written in the form $L-\pi^{-1} L^{\dagger} \pi=2 i[H$, .]. It is shown in [46] that this later definition is equivalent to the previous ones when the Hamiltonian and the Kraus operators are even observables, i.e. $K(H)=H$ and $K\left(V_{i}\right)=V_{i}$.

It was explained in [91] that detailed balance condition is difficult to satisfy for a quantum Markovian system (in particular, it is not satisfied by the usual quantum mechanical damped oscillator). This fact, together with the violation of the usual fluctuation dissipation theorem $[15,67]$ has lead many authors to criticize the Markovian assumption for quantum system. Nevertheless, we emphasize that this assumption is successfully used to model many experimental results. Moreover, it is also possible to find theoretically [5] the generic form of a Markov quantum system for which the Gibbs density $Z^{-1} \exp (-\beta H)$ does obey detailed balance (i.e. $L-\pi^{-1} L^{\dagger} \pi=2 i[H,$.$] ). The set of Kraus operators \left\{V_{i}, i \in[1, \ldots, 2 N]\right\}$ (with $I=2 N$ even) must verify the relation $V_{N-1+i}=\exp \left(-\beta w_{i}\right) V_{i}^{\star}$ for $i \in[1, \ldots, N]$, with $\left[H, V_{i}\right]=-w_{i} V_{i}$.

More generally, the fact that $L^{R}$ defines a bona fide quantum dynamics is a non-trivial fact that imposes stringent conditions on the initial Lindbladian $L[45,46]$. One can readily verify that the stationary density matrix associated with the reversed dynamics is given by $\pi^{R}=K \pi$, because $\left(L^{R}\right)^{\dagger}(K \pi)=0$ (using the fact that $L 1=0$ ).

Finally, we consider a non-stationary set-up with a time-dependent process, governed by a Lindbladian $L_{t}$ and study the process between the initial time $t=0$ and a final time $T$. We wish to consider a reversed process that also runs for times between 0 and $T$. We emphasize that there is not a unique manner to define time-inversion, as was already realized in the case of classical systems [21]. We shall write the time-reversed dynamics by analogy with Eq. (41) and by the requirement that the accompanying distribution of the time-reversed system is the time-reversed of the accompanying distribution of the original system. These two conditions lead to the following Lindbladian:

$$
L_{t^{*}}^{R}=K \pi_{t}^{-1} L_{t}^{\dagger} \pi_{t} K \quad \text { with } t^{*}=T-t .
$$

Here again, $\pi_{t}$ and $\pi_{t}^{-1}$ denote left-multiplication superoperators. This reversal defines a bona fide quantum dynamics if, for example, the initial Lindbladian $L_{t}$ obeys instantaneous detailed balance, $K \pi_{t}^{-1} L_{t}^{\dagger} \pi_{t} K=L_{t}$, because this implies $L_{t^{*}}^{R}=L_{t}$. As shown in $[5,87,88]$, instantaneous detailed balance is satisfied by the Gibbs density $\pi_{t}=$ $Z_{t}^{-1} \exp \left(-\beta H_{t}\right)$ for Markov processes obtained by weak coupling (or low density) limit with a thermal bath at temperature $\beta^{-1}$, that satisfies the KMS condition.

Using Eq. (43), and the relation $L_{t} 1=0$ we find $\left(L_{t^{*}}^{R}\right)^{\dagger} K \pi_{t}=0$. We thus obtain $\pi_{t^{*}}^{R}=$ $K \pi_{t}$, relating, as desired, the accompanying distribution of the time-reversed system with that of the original system. By applying Eqs. (6) and (8) to the time-reversed system, we find that the corresponding evolution superoperator of the time-reversed system is given by $P_{s}^{t, R}=\overrightarrow{\exp }\left(\int_{s}^{t} d u L_{u}^{R}\right)$ and that the multi-time correlations are:

$$
\left\langle\left. O_{1}\left(t_{1}\right) O_{2}\left(t_{2}\right) \ldots O_{N}\left(t_{N}\right)\right|^{R}=\operatorname{Tr}\left(\pi_{0}^{R} P_{0}^{t_{1}, R} O_{1} P_{t_{1}}^{t_{2}, R} O_{2} \ldots P_{t_{N-1}}^{t_{N}, R} O_{N}\right) .\right.
$$


(The superscript $R$ on the 1.h.s. recalls that correlations are taken for the time-reversed process.) We again emphasize that the superoperator $L_{t^{*}}^{R}$, given in Eq. (43), must be a welldefined Lindbladian: this a non-trivial property. This property can be ensured by imposing at each time $t$ the quantum instantaneous detailed balance condition $L_{t^{*}}^{R}=L_{t}$. Here, we do not assume detailed balance and we only require the weaker condition that $L_{t^{*}}^{R}$ is a Lindbladian.

\subsection{Proof of the Quantum Tasaki-Crooks Relation}

Given a scalar $\alpha$, with $0 \leq \alpha \leq 1$, we introduce two $\alpha$-deformed superoperators $L_{t}(\alpha)$ and $L_{t}^{R}(\alpha)$, that act on an observable $X$ as follows:

$$
\begin{aligned}
& L_{t}(\alpha) X=\left(L_{t}+\alpha \pi_{t}^{-1} \partial_{t} \pi_{t}\right) X, \quad \text { and } \\
& L_{t}^{R}(\alpha) X=\left(L_{t}^{R}+\alpha\left(\pi_{t}^{R}\right)^{-1} \partial_{t} \pi_{t}^{R}\right) X .
\end{aligned}
$$

The superoperators $L_{t}(\alpha)$ interpolate between $L_{t}$ (the original Lindbladian) and $L_{t, 1}$ [defined in Eq. (11)] when $\alpha$ varies from 0 to 1 . Similarly, $L_{t}^{R}(\alpha)$ is an interpolation from $L_{t}^{R}$ to $L_{t, 1}^{R}$.

The corresponding $\alpha$-deformed evolution superoperators are given by

$$
P_{s}^{t}(\alpha)=\overrightarrow{\exp }\left(\int_{s}^{t} d u L_{u}(\alpha)\right) \quad \text { and } \quad P_{s}^{t, R}(\alpha)=\overrightarrow{\exp }\left(\int_{s}^{t} d u L_{u}^{R}(\alpha)\right) .
$$

These modified superoperators satisfy the following key duality relation, that lies at the heart of the proof of the quantum fluctuation theorem (for the classical analog in which the dynamics is also modified with respect to a continuous parameter see [63, 69]):

$$
\pi_{0} P_{0}^{T}(\alpha)=\left[\pi_{T} K P_{0}^{T, R}(1-\alpha) K\right]^{\dagger} .
$$

This relation is proved by the differential equation technique. The operator $U_{t}=\pi_{0} P_{0}^{t}(\alpha) \pi_{t}^{-1}$ is equal to 1 at $t=0$ and it satisfies the following evolution equation

$$
\begin{aligned}
\partial_{t} U_{t} & =\pi_{0} P_{0}^{t}(\alpha) \pi_{t}^{-1}\left(\pi_{t} L_{t}(\alpha) \pi_{t}^{-1}-\partial_{t} \pi_{t} \pi_{t}^{-1}\right)=U_{t}\left(\pi_{t} L_{t} \pi_{t}^{-1}+(\alpha-1) \partial_{t} \pi_{t} \pi_{t}^{-1}\right) \\
& =U_{t}\left(K L_{t^{*}}^{R}(1-\alpha) K\right)^{\dagger},
\end{aligned}
$$

where we have used Eq. (43) for the last equality. Hence, we can write

$$
\begin{aligned}
U_{T} & =\overrightarrow{\exp }\left(\int_{0}^{T} d u\left(K L_{T-u}^{R} K\right)^{\dagger}(1-\alpha)\right) \\
& =\left(K \overrightarrow{\exp } \int_{0}^{T} d v L_{v}^{R}(1-\alpha) K\right)^{\dagger}=\left(K P_{0}^{T, R}(1-\alpha) K\right)^{\dagger},
\end{aligned}
$$

which proves Eq. (47).

Applying the duality identity (47) to two arbitrary observables $A$ and $B$ and using the fact that $K$ is multiplicative and anti-unitary, we obtain the relation

$$
\operatorname{Tr}\left(B^{\dagger} \pi_{0} P_{0}^{T}(\alpha) A\right)=\operatorname{Tr}\left(\left(K A^{\dagger}\right) \pi_{0}^{R} P_{0}^{T, R}(1-\alpha)(K B)\right) .
$$

This equation is the essence of the quantum fluctuation theorem and it expresses a generalized detailed balance condition. [Indeed, for a system in a reversible stationary state, i.e. $\pi_{t}=\pi$ and $P_{0}^{T, R}=P_{0}^{T}$, it becomes identical to the detailed balance condition (42) [2, 45, $46,78]$.] Equation (50) can be brought into the following more familiar form by using the same method as in Sect. 3.2 (see Appendix C for more details): 


$$
\begin{aligned}
& \left\langle\left(\pi_{0} B \pi_{0}^{-1}\right)^{\dagger}(0) \overrightarrow{\exp }\left(-\alpha \int_{0}^{T} d u W_{u}(u)\right) A(T)\right\rangle \\
& \quad=\left\langle\left(\pi_{0}^{R}(K A)\left(\pi_{0}^{R}\right)^{-1}\right)^{\dagger}(0) \overrightarrow{\exp }\left(-(1-\alpha) \int_{0}^{T} d u W_{u}^{R}(u)\right)(K B)(T)\right\rangle^{R},
\end{aligned}
$$

where $W_{t}^{R}$ denotes the reversed operator, given by $W_{t}^{R}=-\left(\pi_{t}^{R}\right)^{-1} \partial_{t} \pi_{t}^{R}$. This identity is original and it implies all the other results described in the present work. In particular, if we take $B=\mathbb{1}$ and $\alpha=1$, then Eq. (51) is the quantum Jarzynski-Hatano-Sasa identity (21). If we interpret the mean values in Eq. (51) as classical averages and the operators as commuting c-numbers, then Eq. (51) becomes Crooks' relation.

Remark For a closed system, with an evolution operator $U_{0}^{t}$ given in Eq. (24), we can verify that the time-reversed system (43) is also closed with Hamiltonian $H_{t^{*}}^{R}=K H_{t}$ and evolution operator $U_{0}^{T, R}=K .\left(U_{0}^{T}\right)^{\dagger}$. Then, using identity (47) for $\alpha=1$ and the fact that $K$ is multiplicative, we obtain

$$
\begin{aligned}
P_{0}^{T}(1) A & =\pi_{0}^{-1} K\left(P_{0}^{T, R}\right)^{\dagger} K \pi_{T} A=\pi_{0}^{-1} K U_{0}^{T, R} K\left(\pi_{T} A\right)\left(U_{0}^{T, R}\right)^{\dagger} \\
& =\pi_{0}^{-1} K\left(K\left(\left(U_{0}^{T}\right)^{\dagger}\right) K\left(\pi_{T} A\right) K\left(U_{0}^{T}\right)\right)=\pi_{0}^{-1}\left(U_{0}^{T}\right)^{\dagger} \pi_{T} A U_{0}^{T} .
\end{aligned}
$$

Substituting the last expression in Eq. (50) leads to

$$
\operatorname{Tr}\left(B^{\dagger} \pi_{0} \pi_{0}^{-1} U_{0}^{T \dagger} \pi_{T} A U_{0}^{T}\right)=\operatorname{Tr}\left(K\left(A^{\dagger}\right) \pi_{0}^{R}\left(U_{0}^{T, R}\right)^{\dagger} K(B) U_{0}^{T, R}\right) .
$$

Recalling that $\pi_{0}^{-1}$ and $\pi_{T}$ are given by the Boltzmann law, the above equation becomes in the Heisenberg representation denoted by the superscript $\mathcal{H}$,

$$
\begin{aligned}
& \operatorname{Tr}\left(B^{\dagger} \pi_{0} \exp \left(\beta H_{0}^{\mathcal{H}}(0)\right) \exp \left(-\beta H_{T}^{\mathcal{H}}(T)\right) A^{\mathcal{H}}(T)\right) \\
& =\frac{Z_{T}}{Z_{0}} \operatorname{Tr}\left(K\left(A^{\dagger}\right) \pi_{0}^{R}\left(U_{0}^{T, R}\right)^{\dagger} K(B) U_{0}^{T, R}\right) .
\end{aligned}
$$

We emphasize that for $B=\mathbb{1}, \mathrm{Eq}(53)$ is a tautology (because $K$ is anti-unitary), however it implies the non-trivial result (54): this feature is characteristic of most of the derivations of the work identities. If we take $A=B=\mathbb{1}$, we retrieve the quantum Jarzynski relation for closed systems as first found by Kurchan and Tasaki $[68,95]$.

\section{Conclusion}

In this work, we have derived fluctuations relations for an open quantum system described by a Lindblad dynamics that takes into account the interactions with the environment as well as measurement processes. We prove the fluctuations relations thanks to a suitable deformation of the system's dynamics: this crucial technical idea provides a truly unified picture of the fluctuations relations, whether classical or quantum, and does not require to define the concept of work at the quantum level. The Markovian hypothesis that underlies the Lindblad dynamics is a strong assumption $[83,91]$. Besides, the linearity of the quantum map is not necessarily guaranteed for initial states that do not factorize [47] and one must impose strong conditions such as the absence of initial correlations between the system and the environment [72]. (Note that the article [73] gives more general conditions that allow a subdynamics to be a good approximation of the exact evolution even if the initial systemreservoir state is correlated.) However, despite its flaws, this Markovian model allows us to derive precise mathematical results that are prone to experimental verifications $[19,59]$. 
Quantum Fluctuation Relations for open systems are, at present, not as developed as their classical counterparts. One major difficulty in the quantum realm is the lack of a trajectory picture when coherence and measurements are taken into account. To overcome this difficulty, an unraveling of the Lindblad equation $[13,52]$ must be used. Previous attempts of this idea were performed in $[32,35,36,58]$ and we plan to extend the results of the present work by using such unravelings [8]. Another possible extension of our work would be to study the effect of choosing a time inversion different from that of Eq. (43): this may lead to various families of fluctuation relations, as happened in the classical case [21]. One could also study particles with non-zero spins such as Dirac spinors. Exact solutions of specific models, such as quantum Brownian motion solution of the Markovian (non Lindblad) Caldeira-Leggett equation [14], may also provide us with experimentally testable predictions. More precisely, the formal relation between the classical exclusion process and the Lindblad evolution of free fermions in one dimension [40,96], could allow us to use, for open quantum systems, the exact results obtained for the large deviation functions of various stochastic processes $[33,76]$. Finally, the investigation of time-reversal properties of quantum Non-Markovian systems [48], in which the characteristic time scale of the environment can not be neglected w.r.t. that of the system, should also yield interesting fluctuation relations.

Acknowledgements R.C. thanks K. Gawędzki for pointing out the fact that the Lindbladian character of Eq. (43) is non-trivial and the relation with detailed balance. R.C. acknowledges the support of the Koshland center for basic research. K.M. thanks M. Bauer and H. Orland for useful comments and S. Mallick for useful remarks on the manuscript. Results similar to those presented here were also reached independently by K. Gawędzki and S. Attal some time ago [7].

\section{Appendix A: Proof of Eq. (30)}

The identity (30) is proved by using the differential equation technique. First, we note that both sides of Eq. (30) coincide at $t=0$. Then, we find that the time derivative of the 1.h.s. is given by

$$
\frac{d}{d t}\left\{\overrightarrow{\exp }\left(-\int_{0}^{t} W_{u}(u)^{\mathcal{H}} d u\right)\right\}=\overrightarrow{\exp }\left(-\int_{0}^{t} W_{u}(u)^{\mathcal{H}} d u\right)\left(-W_{t}(t)^{\mathcal{H}}\right) .
$$

This follows from the very definition of a time-ordered exponential.

The time derivative of the r.h.s. is given by

$$
\begin{aligned}
\frac{d}{d t}\left(\left(\pi_{0}\right)^{-1} \pi_{t}^{\mathcal{H}}(t)\right) & =\left(\pi_{0}\right)^{-1} \frac{d}{d t}\left\{\left(U_{0}^{t}\right)^{\dagger} \pi_{t} U_{0}^{t}\right\}=\left(\pi_{0}\right)^{-1}\left\{i\left(U_{0}^{t}\right)^{\dagger}\left[H_{t}, \pi_{t}\right] U_{0}^{t}+\left(\frac{d \pi_{t}}{d t}\right)^{\mathcal{H}}\right\} \\
& =\left(\pi_{0}\right)^{-1} \pi_{t}^{\mathcal{H}}(t)\left\{\left(\pi_{t}^{\mathcal{H}}(t)\right)^{-1}\left(\frac{d \pi_{t}}{d t}\right)^{\mathcal{H}}\right\} \\
& =\left(\pi_{0}\right)^{-1} \pi_{t}^{\mathcal{H}}(t)\left(-W_{t}(t)^{\mathcal{H}}\right)
\end{aligned}
$$

where we have used the fact that $\pi_{t}$ commutes with $H_{t}$. The last equality follows from the definition (10) of $W_{t}$. We have thus shown that the 1.h.s. and the r.h.s coincide at $t=0$ and that they satisfy the same first order differential with respect to time: they are therefore identical for all times. 


\section{Appendix B: Proof of the Callen-Welton-Kubo Formula Using the Steady-State Quantum Fluctuation Dissipation Theorem (39)}

In this appendix, we show that the steady-state quantum Fluctuation Dissipation Theorem (39) is equivalent, in the case of a closed system perturbed near equilibrium, to the Callen-Welton-Kubo relation [15, 67].

We start with a time-independent Hamiltonian $H_{0}$ with invariant density-matrix given by $\pi_{0}=Z_{0}^{-1} \exp \left(-\beta H_{0}\right)$. We then consider a perturbation of $H_{0}$ of the form

$$
H_{t}=H_{0}-h^{a}(t) O_{a} .
$$

In a closed system, the Lindblad equation reduces to the Liouville equation

$$
\partial_{t} \rho_{t}=L_{t}^{\dagger} \rho_{t}=-i\left[H_{t}, \rho_{t}\right] \text {. }
$$

The accompanying density is explicitly given by $\pi_{t}=Z_{t}^{-1} \exp \left(-\beta H_{t}\right)$. Comparing with Eq. (35), we find $M_{a}=i\left[O_{a},.\right]$.

We now derive an explicit expression for the operators $D_{a}$ defined in Eq. (38). Starting with the exact formula

$$
\exp \left(-\beta H_{t}\right)=\overrightarrow{\exp }\left(h_{t}^{a} \int_{0}^{\beta} d \alpha \exp (-\alpha H) O_{a} \exp (\alpha H)\right) \exp (-\beta H)
$$

(which can be proved by differentiating both sides w.r.t. $\beta$ ), we find at first order [82]

$$
\exp \left(-\beta H_{t}\right)=\exp (-\beta H)+h_{t}^{a} \int_{0}^{\beta} d \alpha \exp (-\alpha H) O_{a} \exp (\alpha H) \exp (-\beta H)+o(h) .
$$

This implies that

$$
\begin{aligned}
Z_{t} & =\operatorname{Tr}(\exp (-\beta H))+h_{t}^{a} \beta \operatorname{Tr}\left(O_{a} \exp (-\beta H)\right)+o(h) \\
& =Z\left(1+h_{t}^{a} \beta\left\langle O_{a}\right\rangle_{\pi_{0}}\right)+o(h) .
\end{aligned}
$$

The first order perturbation of $W_{t}$ is then given by

$$
W_{t}=\dot{h}_{t}^{a}\left(\beta\left\langle O_{a}\right\rangle_{\pi_{0}}-\exp (+\beta H) \int_{0}^{\beta} d \alpha \exp (-\alpha H) O_{a} \exp (\alpha H) \exp (-\beta H)\right)+o(h) .
$$

Comparing with Eq. (38), we obtain the analytical expression for $D_{a}$ :

$$
D_{a}=-\beta\left\langle O_{a}\right\rangle_{\pi_{0}}+\int_{0}^{\beta} d \alpha \exp (\alpha H) O_{a} \exp (-\alpha H) .
$$

We now transform, using (8), the r.h.s. of the quantum Fluctuation Dissipation Theorem (39) as follows:

$$
\begin{aligned}
\frac{d}{d u}\left\langle D_{a}(u) A(t)\right\rangle_{\pi_{0}} & =\frac{d}{d u} \operatorname{Tr}\left(\pi_{0} D_{a} P_{u}^{t} A\right)=-\operatorname{Tr}\left(\pi_{0} D_{a} L P_{u}^{t} A\right) \\
& =-\left(D_{a}^{\dagger} \pi_{0}, L P_{u}^{t} A\right)=-\left(L^{\dagger}\left(D_{a}^{\dagger} \pi_{0}\right), P_{u}^{t} A\right) \\
& =-\operatorname{Tr}\left(\left(L^{\dagger}\left(D_{a}^{\dagger} \pi_{0}\right)\right)^{\dagger} P_{u}^{t} A\right)=-\operatorname{Tr}\left(\pi_{0} \pi_{0}^{-1}\left(L^{\dagger}\left(\pi_{0} D_{a}\right)\right) P_{u}^{t} A\right) .
\end{aligned}
$$

Note that in the first equality, we use the fact that $\pi_{0}$ is the invariant density of the unperturbed dynamics. The quantum Fluctuation Dissipation Theorem (39) can thus be rewritten as

$$
\left.\frac{\delta\langle A(t)\rangle}{\delta h^{a}(u)}\right|_{h=0}=\left\langle E_{a}(u) A(t)\right\rangle_{\pi_{0}}
$$


where we have defined

$$
E_{a}=-\pi_{0}^{-1} L^{\dagger}\left(\pi_{0} D_{a}\right)
$$

From Eq. (B.7), we deduce the analytical expression of $E_{a}$ :

$$
\begin{aligned}
E_{a} & =-\pi_{0}^{-1} L^{\dagger}\left(\pi_{0} D_{a}\right) \\
& =i \exp (\beta H)\left[H, \exp (-\beta H) \int_{0}^{\beta} d \alpha \exp (\alpha H) O_{a} \exp (-\alpha H)\right] \\
& =i\left[H, \int_{0}^{\beta} d \alpha \exp (\alpha H) O_{a} \exp (-\alpha H)\right]=i \int_{0}^{\beta} d \alpha \exp (\alpha H)\left[H, O_{a}\right] \exp (-\alpha H) \\
& =i \int_{0}^{\beta} d \alpha \frac{d}{d \alpha}\left(\exp (\alpha H) O_{a} \exp (-\alpha H)\right)=i \exp (\beta H) O_{a} \exp (-\beta H)-i O_{a} .
\end{aligned}
$$

We remark that the terms on the r.h.s. can be interpreted as the analytic continuation in imaginary time (as allowed by the KMS condition [77]) of the Heisenberg representation with respect to the unperturbed Hamiltonian $H$. Thus, we have

$$
E_{a}=i O_{a}^{H}(-i \beta)-i O_{a}^{H}(0) .
$$

Finally, (B.8) becomes for $(u<T)$

$$
\begin{aligned}
\left.\frac{\delta\langle A(t)\rangle}{\delta h^{a}(u)}\right|_{h=0} & =i \operatorname{Tr}\left(\pi_{0} O_{a}^{H}(-i \beta) A^{H}(t-u)\right)-i \operatorname{Tr}\left(\pi_{0} O_{a}^{H}(0) A^{H}(t-u)\right) \\
& =i\left\langle\left. O_{a}(-i \beta) A(t-u)\right|_{\pi_{0}}-i\left\langle O_{a}(0) A(t-u)\right\rangle_{\pi_{0}}\right. \\
& =i\left\langle O_{a}(0) A(t-u+i \beta)\right\rangle_{\pi_{0}}-i\left\langle O_{a}(0) A(t-u)\right\rangle_{\pi_{0}} .
\end{aligned}
$$

The last equality follows from the fact that the correlation $\left\langle X_{s} Y_{t}\right\rangle_{\pi_{0}}$ depends just on $t-s$. This equation is the real space version of the Callen-Welton-Kubo equation. The more conventional form is obtained by performing a Fourier Transform with respect to time. The susceptibility is defined as (using causality, i.e. $\left.\frac{\delta\langle A(t)\rangle}{\delta h^{a}(u)}\right|_{h=0}=0$ if $u>t$ )

$$
\begin{aligned}
\chi_{O_{a} A}(w) & =\left.\int_{0}^{\infty} d t \frac{\delta\langle A(t)\rangle}{\delta h^{a}(0)}\right|_{h=0} \exp (i w t) \\
& =i(\exp (\beta w)-1) \int_{0}^{\infty} d t\left\langle O_{a}(0) A(t)\right\rangle_{0} \exp (i w t)
\end{aligned}
$$

where we have used Eq. (B.11) in the last equality. The symmetrized correlation can be written as

$$
\begin{aligned}
C_{O_{a} A}^{S}(w)= & \frac{1}{2} \int_{-\infty}^{+\infty} d t \exp (i w t) \operatorname{Tr}\left(\pi_{0} O_{a}^{H}(0) A^{H}(t)\right) \\
& +\frac{1}{2} \int_{-\infty}^{+\infty} d t \exp (i w t) \operatorname{Tr}\left(\pi_{0} A^{H}(t) O_{a}^{H}(0)\right) \\
= & \frac{1}{2} \int_{0}^{+\infty} d t \exp (i w t) \operatorname{Tr}\left(\pi_{0} O_{a}^{H}(0) A^{H}(t)\right) \\
& +\frac{1}{2} \int_{0}^{+\infty} d t \exp (-i w t) \operatorname{Tr}\left(\pi_{0} O_{a}^{H}(0) A^{H}(-t)\right) \\
& +\frac{1}{2} \int_{0}^{+\infty} d t \exp (i w t) \operatorname{Tr}\left(\pi_{0} A^{H}(t) O_{a}^{H}(0)\right)
\end{aligned}
$$




$$
\begin{aligned}
& +\frac{1}{2} \int_{0}^{+\infty} d t \exp (-i w t) \operatorname{Tr}\left(\pi_{0} A^{H}(-t) O_{a}^{H}(0)\right) \\
= & \frac{1}{2} \int_{0}^{+\infty} d t \exp (i w t) \operatorname{Tr}\left(\pi_{0} O_{a}^{H}(0) A^{H}(t)\right) \\
& +\frac{1}{2} \int_{0}^{+\infty} d t \exp (-i w t) \operatorname{Tr}\left(\pi_{0} A^{H}(0) O_{a}^{H}(t+i \beta)\right) \\
& +\frac{1}{2} \int_{0}^{+\infty} d t \exp (i w t) \operatorname{Tr}\left(\pi_{0} O_{a}^{H}(0) A^{H}(t+i \beta)\right) \\
& +\frac{1}{2} \int_{0}^{+\infty} d t \exp (-i w t) \operatorname{Tr}\left(\pi_{0} A^{H}(0) O_{a}^{H}(t)\right) \\
= & \frac{1}{2}(1+\exp (\beta w)) \int_{0}^{\infty} d t\left\langle O_{a}(0) A(t)\right\rangle_{0} \exp (i w t) \\
& +\frac{1}{2}(1+\exp (-\beta w)) \int_{0}^{\infty} d t\left\langle A(0) O_{a}(t)\right\rangle_{0} \exp (-i w t),
\end{aligned}
$$

where we have used a KMS type identity to obtain the third equality: $\operatorname{Tr}\left(\pi_{0} X^{H}(s) Y^{H}(t)\right)=$ $\operatorname{Tr}\left(\pi_{0} Y^{H}(t) X^{H}(s+i \beta)\right)$. By using (B.12) and (B.13), we obtain

$$
\begin{aligned}
C_{O_{a} A}^{S}(w) & =\frac{1}{2}(1+\exp (\beta w)) \frac{\chi_{O_{a} A}(w)}{i(\exp (\beta w)-1)}+\frac{1}{2}(1+\exp (-\beta w)) \frac{\chi_{A O_{a}}(-w)}{i(\exp (-\beta w)-1)} \\
& =\frac{\operatorname{coth}\left(\frac{\beta w}{2}\right)}{2 i}\left(\chi_{O_{a} A}(w)-\chi_{A O_{a}}(-w)\right) .
\end{aligned}
$$

Note that in the expression for $\chi_{A O_{a}}$, the operator $A$ is considered to be the perturbation and $O_{a}$ the observable. We thus implicitly suppose that $A=A^{\dagger}$. In particular, if we take $A=O_{b}$

$$
\begin{aligned}
C_{O_{a} O_{b}}^{S}(w) & =\frac{\operatorname{coth}\left(\frac{\beta w}{2}\right)}{2 i}\left(\chi_{o_{a} O_{b}}(w)-\chi_{O_{b} O_{a}}(-w)\right) \\
& =\operatorname{coth}\left(\frac{\beta w}{2}\right) \operatorname{Im}\left(\chi_{O_{a} O_{b}}(w)\right),
\end{aligned}
$$

recalling that $\chi_{A O_{a}}(-w)=\overline{\chi_{A O_{a}}(w)}$. For a closed system, an alternative proof showing that relation (31) implies Eq. (B.15) is given in [9].

\section{Appendix C: Derivation of Eq. (51)}

First, we establish the following relation, valid for two operators $X$ and $Y$

$$
\operatorname{Tr}\left(\pi_{0} Y P_{0}^{T}(\alpha) X\right)=\left\langle Y(0) \overrightarrow{\exp }\left(-\alpha \int_{0}^{T} d u W_{u}(u)\right) X(T)\right\rangle .
$$

The method to derive this formula is identical to the one used in Eqs. (16) to (20): we perform Dyson-Schwinger expansion $P_{0}^{T}(\alpha)$ w.r.t. the deformation parameter $\alpha$, rewrite the trace as a correlation function via Eq. (8) and the result is resummed as a time-ordered exponential. We recall that $W_{t}$ was defined in Eq. (10). The same equation is true for the reversed system, with $\alpha$ replaced by $1-\alpha$ and where the reversed 'injected-power' operator is given by $W_{t}^{R}=-\left(\pi_{t}^{R}\right)^{-1} \partial_{t} \pi_{t}^{R}$ :

$$
\operatorname{Tr}\left(\pi_{0} Y P_{0}^{R, T}(1-\alpha) X\right)=\left\langle Y(0) \overrightarrow{\exp }\left(-(1-\alpha) \int_{0}^{T} d u W_{u}^{R}(u)\right) X(T)\right\rangle^{R} .
$$


Replacing both sides of Eq. (50) by the relations (C.1) and (C.2) and inserting the duality identity (47) into Eq. (C.1), leads to the Fluctuation Relation (51) for an open quantum Markovian system.

\section{References}

1. Accardi, L.: On the quantum Feynman-Kac formula. Milan J. Math. 48(1), 135-180 (1978)

2. Agarwal, G.S.: Open quantum Markovian systems and the microreversibility, Z. Phys. 258, 409 (1972)

3. Alicki, R.: On the detailed balance condition for non-Hamiltonian systems. Rep. Math. Phys. 10, 2249 (1976)

4. Alicki, R.: The quantum open system as a model of the heat engine. J. Phys. A 12, 5 (1979)

5. Alicki, R., Lendi, K.: Quantum Dynamical Semigroups and Applications. Lecture Notes Phys., vol. 717. Springer, Berlin (2007)

6. Allahverdyan, A.E., Nieuwenhuizen, T.M.: Fluctuations of works from quantum sub-ensembles: the case against quantum work-fluctuation theorems. Phys. Rev. E 71, 066102 (2005)

7. Attal, S., Gawędzki, K.: Private communication

8. Attal, S., Joye, A., Pillet, C.A.: Quantum Open Systems. Vol. II: The Markovian Approach. Lecture Notes in Mathematics, vol. 1881. Springer, Berlin (2006)

9. Andrieux, D., Gaspard, P.: Quantum work relations and response theory. Phys. Rev. Lett. 100, 230404 (2008)

10. Andrieux, D., Gaspard, P., Monnai, T., Tasaki, S.: Fluctuation theorem for currents in open quantum systems. New J. Phys. 11, 043014 (2009)

11. Bauer, M., Bernard, D.: Quantum stochastic processes: a case study. J. Stat. Mech. P04016 (2011)

12. Bochkov, G.N., Kuzovlev, Y.E.: On general theory of thermal fluctuations in non linear systems. Sov. Phys. JETP 45, 125-130 (1977)

13. Breuer, H.P., Petruccione, F.: The Theory of Open Quantum Systems. Oxford University Press, London (2002)

14. Caldeira, A.O., Leggett, A.J.: Influence of damping on quantum interference: an exactly soluble model. Phys. Rev. A 31, 1057 (1985)

15. Callen, H.B., Welton, T.A.: Irreversibility and generalized noise. Phys. Rev. 83, 34 (1951)

16. Callens, I., De Roeck, W., Jacobs, T., Maes, C., Netocny, K.: Quantum entropy production as a measure of irreversibility. Physica D 187(1-4), 11 (2002).

17. Campisi, M., Talkner, P., Hänggi, P.: Fluctuation theorem for arbitrary open quantum systems. Phys. Rev. Lett. 102, 210401 (2009)

18. Campisi, M., Talkner, P., Hänggi, P.: Quantum Bochkov-Kuzovlev work fluctuation theorems. Philos. Trans. R. Soc. Lond. A 369, 291 (2011)

19. Campisi, M., Talkner, P., Hänggi, P.: Quantum fluctuation relations: foundations and applications. Rev. Mod. Phys. 83, 771 (2011)

20. Chernyak, V., Mukamel, S.: Effect of quantum collapse on the distribution of work in driven single molecules. Phys. Rev. Lett. 93, 048302 (2004)

21. Chetrite, R., Gawȩdzki, K.: Fluctuation relations for diffusion processes. Commun. Math. Phys. 282, 469 (2008)

22. Chetrite, R., Falkovich, G., Gawȩdzki, K.: Fluctuation relations in simple examples of non-equilibrium steady states. J. Stat. Mech. P08005 (2008)

23. Crooks, G.E.: Nonequilibrium measurements of free energy differences for microscopically reversible Markovian systems. J. Stat. Phys. 90, 1481 (1999)

24. Crooks, G.E.: Entropy production fluctuation theorem and the nonequilibrium work relation for free energy differences. Phys. Rev. E 60, 2721 (1999)

25. Crooks, G.E.: On the quantum Jarzynski identity. J. Stat. Mech. P10023 (2008)

26. Crooks, G.E.: Quantum operation time reversal. Phys. Rev. A 77, 034101 (2008)

27. Cohen-Tannoudji, C., Dupont-Roc, J., Grynberg, G.: Photons and Atoms. Wiley, New York (1992)

28. Davies, E.B.: Quantum Theory of Open Systems. Academic Press, San Diego (1976)

29. Deffner, S., Lutz, E.: Nonequilibrium entropy production for open quantum systems. Phys. Rev. Lett. 107, 140404 (2011)

30. DelMoral, P.: Feynman-Kac Formulae Genealogical and Interacting Particle Systems with Applications. Probability and Applications. Springer, New York (2004)

31. Derezinski, J., De Roeck, W.: Extended weak coupling limit for Pauli-Fierz operators. Commun. Math. Phys. 279, 1-30 (2008) 
32. Derezinski, J., De Roeck, W., Maes, C.: Fluctuations of quantum currents and unravelings of master equations. J. Stat. Phys. 131, 341 (2008)

33. Derrida, B.: Non-Equilibrium steady states: fluctuations and large deviations of the density and the current. J. Stat. Mech. P07023 (2007)

34. De Roeck, W., Maes, C.: Quantum version of free-energy-irreversible-work relations. Phys. Rev. E 69, 026115 (2004)

35. De Roeck, W., Maes, C.: Fluctuations of the dissipated heat for a quantum stochastic model. Rev. Math. Phys. 18, 619 (2006)

36. De, W.: Roeck quantum fluctuation theorem: can we go from micro to meso. C. R. Phys. 8, 674 (2007)

37. Douarche, F., Ciliberto, S., Petrosyan, A., Rabbiosi, I.: An experimental test of the Jarzynski equality in a mechanical experiment. Europhys. Lett. 70, 593 (2005)

38. Dumcke, R., Spohn, H.: The proper form of the generator in the weak coupling limit. Z. Phys. B 34, 419-422 (1979)

39. Dumcke, R.: The low density limit for an N-level system interacting with a free Bose or Fermi gas. Commun. Math. Phys. 97, 331 (1985)

40. Eisler, V.: Crossover between ballistic and diffusive transport: the quantum exclusion process. J. Stat. Mech. P06007 (2011)

41. Engel, A., Nolte, R.: Jarzynski equation for a simple quantum system: comparing two definitions of work. Europhys. Lett. 79, 10003 (2007)

42. Esposito, M., Mukamel, S.: Fluctuation theorems for quantum master equations. Phys. Rev. E 73, 046129 (2006)

43. Esposito, M., Harbola, U., Mukamel, S.: Nonequilibrium fluctuations, fluctuation theorems, and counting statistics in quantum systems. Rev. Mod. Phys. 81, 1665 (2009)

44. Evans, D.J., Cohen, E.G.D., Morriss, G.P.: Probability of second law violations in shearing steady states. Phys. Rev. Lett. 71, 2401 (1993)

45. Fagnola, F., Umanità, V.: Generators of detailed balance quantum Markov semigroups. Infin. Dimens. Anal. Quantum Probab. Relat. Top. 10, 335 (2007). arXiv:0707.2147v2 [math-ph]

46. Fagnola, F., Umanità, V.: Detailed balance, time reversal and generators of quantum Markov semigroups. Math. Notes (Mat. Zametki) 84, 108 (2008)

47. Fonseca Romero, K.M., Talkner, P., Hänggi, P.: Is the dynamics of open quantum systems always linear? Phys. Rev. A 69, 052109 (2004)

48. Ford, G.W., Connell, R.F.: There is no quantum regression theorem. Phys. Rev. Lett. 77, 5 (1996)

49. Frigerio, A., Gorini, V., Kossakowski, A., Verri, M.: Quantum detailed balance and KMS condition. Commun. Math. Phys. 57(2), 97-110 (1977)

50. Frigerio, A., Gorini, V.: Markov dilations and quantum detailed balance. Commun. Math. Phys. 93(4), 517-532 (1984)

51. Gallavotti, G., Cohen, E.G.D.: Dynamical ensembles in nonequilibrium statistical mechanics. Phys. Rev. Lett. 74, 2694 (1995)

52. Gardiner, C.W., Zoller, P.: Quantum Noise. Springer, Berlin (2000)

53. Gorini, V., Kossakowski, A.: $N$-level system in contact with a singular reservoir. J. Math. Phys. 17, 7 (1976).

54. Hänggi, P., Thomas, H.: Stochastic processes: time evolution, symmetries and linear response. Phys. Rep. 88, 207 (1982)

55. Haroche, S., Raymond, J.-M.: Exploring the Quantum. Oxford University Press, London (2006)

56. Hatano, T., Sasa, S.: Steady-state thermodynamics of Langevin systems. Phys. Rev. Lett. 86, 3463-3466 (2001)

57. Hepp, K., Lieb, H.: Phase transition in reservoir driven open systems with applications to lasers and superconductors. Helv. Phys. Acta 46, 573-602 (1973)

58. Horowitz, J.M.: Quantum trajectory approach to the stochastic thermodynamics of a forced harmonic oscillator. Phys. Rev. E 85, 031110 (2012)

59. Huber, G., Schmidt-Kaler, F., Deffner, S., Lutz, E.: Employing trapped cold ions to verify the quantum Jarzynski equality. Phys. Rev. Lett. 101, 070403 (2008)

60. Hummer, G., Szabo, A.: Free energy reconstruction from nonequilibrium single-molecule pulling experiments. Proc. Natl. Acad. Sci. USA 98, 3658 (2001)

61. Komnik, A., Saleur, H.: Quantum fluctuation theorem in an interacting setup: point contacts in fractional quantum hall edge state devices. Phys. Rev. Lett. 107, 100601 (2011)

62. Jarzynski, C.: Nonequilibrium equality for free energy differences. Phys. Rev. Lett. 78, 2690 (1997)

63. Jarzynski, C.: Equilibrium free-energy differences from nonequilibrium measurements: a masterequation approach. Phys. Rev. E 56, 5018 (1997)

64. Jarzynski, C., Wójcik, D.K.: Classical and quantum fluctuation theorems for heat exchange. Phys. Rev. Lett. 92, 230602 (2004) 
65. Klich, I.: Full counting statistics, an elementary derivation of Levitov's formula. In: Nazarov, Yu.V., Blanter, Y.M., (eds.) Quantum Noise. Kluwer, Dordrecht (2003)

66. Kubo, R.: The fluctuation-dissipation theorem. Rep. Prog. Phys. 29, 255-284 (1966)

67. Kubo, R., Toda, M., Hashitsume, N.: Statistical Physics II: Nonequilibrium Statistical Physics. Springer, Berlin (1998)

68. Kurchan, J.: A quantum fluctuation theorem. arXiv:cond-mat/0007360 (2000)

69. Kurchan, J.: Fluctuation theorem for stochastic dynamics. J. Phys. A, Math. Gen. 31, 3719 (1998)

70. Lebowitz, J.L., Spohn, H.: A Gallavotti-Cohen-type symmetry in the large deviation functional for stochastic dynamics. J. Stat. Phys. 95, 333 (1999)

71. Lindblad, G.: On the generators of quantum dynamical semigroups. Commun. Math. Phys. 48, 119 (1976)

72. Lindblad, G.: On the existence of quantum subdynamics. J. Phys. A 29, 4197-4207 (1996)

73. Lindblad, G.: On the existence of quantum subdynamics. J. Math. Phys. 39, 5 (1998)

74. Liphardt, J., Dumont, S., Smith, S.B., Tinoco, I., Bustamante, C.: Equilibrium information from nonequilibrium measurements in an experimental test of Jarzynski's equality. Science 7(296), 5574 (2002)

75. Liu, F.: A derivation of quantum Jarzynski equality using quantum Feyman-Kac formula. arXiv:1201.1557 (2012)

76. Mallick, K.: Some exact results for the exclusion process. J. Stat. Mech. P01024 (2011)

77. Martin, P.C., Schwinger, J.: Theory of many-particle systems. I. Phys. Rev. 115, 1342 (1959)

78. Majewski, W.A.: The detailed balance condition in quantum statistical mechanics. J. Math. Phys. 25, 614 (1984)

79. Monnai, T.: Unified treatment of the quantum fluctuation theorem and the Jarzynski equality in terms of microscopic reversibility. Phys. Rev. E 72, 027102 (2005)

80. Putz, W., Woronowicz, S.L.: Passive states and KMS states for general quantum systems. Commun. Math. Phys. 58, 273-290 (1978)

81. Mukamel, S.: Quantum extension of the Jarzynski relation: analogy with stochastic dephasing. Phys. Rev. Lett. 90, 170604 (2003)

82. Negele, J.W., Orland, H.: Quantum Many-Particle Systems. Westview, Boulder (1988)

83. Pechukas, P.: Reduced dynamics need not be completely positive. Phys. Rev. Lett. 73, 8 (1994)

84. Prost, J., Joanny, J.-F., Parrondo, J.M.R.: Generalized fluctuation-dissipation theorem for steady-state systems. Phys. Rev. Lett. 103, 090601 (2009)

85. Saito, K., Dhar, A.: Fluctuation theorem in quantum heat conduction. Phys. Rev. Lett. 99, 180601 (2007)

86. Saito, K., Utsumi, Y.: Symmetry in full counting statistics, fluctuation theorem, and relations among nonlinear transport coefficients in the presence of a magnetic field. Phys. Rev. B 78, 115429 (2008)

87. Spohn, H.: Entropy production for quantum dynamical semigroups. J. Math. Phys. 19, 5 (1978)

88. Spohn, H., Lebowitz, J.L.: Irreversible thermodynamics for quantum systems weakly coupled to thermal reservoirs. Adv. Chem. Phys. 38, 109 (1978)

89. Stratonovich, R.L.: Nonlinear Nonequilibrium Thermodynamics II. Springer, Berlin (1994)

90. Schiff, L.I.: Quantum Mechanics. McGraw-Hill, New York (1968)

91. Talkner, P.: The failure of the quantum regression hypothesis. Ann. Phys. 167, 390-436 (1986)

92. Talkner, P., Lutz, E., Hänggi, P.: Fluctuation theorem: work is not an observable. Phys. Rev. E 75, 050102(R) (2007)

93. Talkner, P., Hänggi, P.: The Tasaki-Crooks quantum fluctuation theorem. J. Phys. A 40, F569 (2007)

94. Talkner, P., Campisi, M., Hänggi, P.: Fluctuation theorems in driven open quantum systems. J. Stat. Mech. P02025 (2009)

95. Tasaki, H.: Jarzynski relations for quantum systems and some applications. arXiv:cond-mat/0009244 (2000)

96. Temme K, K., Wolf, M.M., Verstraete, F.: Stochastic exclusion processes versus coherent transport. arXiv:0912.0858

97. Weidlich, W.: Fluctuation-dissipation theorem for a class of stationary open systems. Z. Phys. 248, 234 (1971)

98. Yukawa, S.: A quantum analogue of the Jarzynski equality. J. Phys. Soc. Jpn. 69, 2367 (2000) 